\title{
Disfunción diafragmática inducida por ventilación mecánica
}

\author{
JORGE VALENZUELA V. ${ }^{1}$, RAMÓN PINOCHET U. ${ }^{2}$, MÁXIMO ESCOBAR C. ${ }^{2}$, \\ JOSÉ LUIS MÁRQUEZ A. ${ }^{2}$, RAQUEL RIQUELME V. ${ }^{1}$, PABLO CRUCES R. ${ }^{1,3}$ \\ 1. Unidad de Paciente Crítico Pediátrico. Hospital El Carmen de Maipú. \\ 2. Escuela de Kinesiología. Universidad Católica del Maule. \\ 3. Centro de Investigación de Medicina Veterinaria, Escuela de Medicina Veterinaria, Facultad de Ecología y Recursos \\ Naturales, Universidad Andres Bello. \\ Centro de Investigación de Medicina Veterinaria, Universidad Andrés Bello.
}

\begin{abstract}
\section{Ventilator-induced Diaphragmatic dysfunction}

The ventilator-induced diaphragmatic dysfunction is a group of disorders caused by the inactivity of the diaphragm muscle during controlled mechanical ventilation. From a structural point of view, the diaphragm subjected to mechanical ventilation undergoes an atrophy process, in which decreased synthesis and increased muscle protein degradation are observed. Recent research has identified different molecular signaling pathways that link both processes in the diaphragm, describing compelling evidence that shows that oxidative stress contributes to this phenomenon. Also, functional changes characterized by a reduction in stress and fatigue resistance, associated with an increase in the maximum shortening velocity are observed. The purpose of the present review is to analyze the impact of mechanical ventilation on the structure and function of the diaphragm muscle. Clinical implications and potential preventive steps are discussed. Since the duration of mechanical ventilation is the primary responsible for the absence of mechanical stimulation on the diaphragm, the use of ventilatory strategies for successful early weaning and early use of partial modalities should be the main pillars in the prevention of this condition.
\end{abstract}

(Key words: Mechanical ventilation, diaphragm, muscle atrophy, oxidative stress).

Rev Chil Pediatr 2014; 85 (4): 491-498

\section{RESUMEN}

La disfunción diafragmática inducida por ventilación mecánica consiste en un conjunto de alteraciones producidas por la inactividad del músculo diafragma durante la ventilación mecánica controlada. A nivel estructural, el diafragma sometido a ventilación mecánica experimenta un proceso de atrofia por desuso, en el que se

Recibido el 15 de julio de 2014, última versión aceptada para publicación el 18 de agosto de 2014.

\section{Potenciales conflictos de interés:}

Estudio financiado por Concurso Interno Universidad Católica del Maule 2012.

Los autores declaran no tener potenciales conflictos de interés.

Este trabajo cumple con los requisitos sobre consentimiento /asentimiento informado, comité de ética, financiamiento, estudios animales y sobre la ausencia de conflictos de intereses según corresponda. 
observa una disminución de la síntesis y aumento en la degradación de proteínas musculares. Recientemente se han identificado diferentes rutas de señalización molecular que vinculan ambos procesos en el diafragma, existiendo evidencia fehaciente que el estrés oxidativo contribuye a este fenómeno. Concomitantemente, se han observado cambios funcionales caracterizados por una reducción de la fuerza y resistencia a la fatiga, asociado a un incremento en la velocidad máxima de acortamiento. El objetivo principal de esta revisión es analizar el impacto de la ventilación mecánica sobre la estructura y función del músculo diafragma. Además se discuten implicancias clínicas y potenciales intervenciones preventivas para esta emergente entidad. Dado que el tiempo de permanencia en ventilación mecánica controlada es el principal factor responsable de la ausencia de estímulo mecánico sobre el diafragma, el empleo de estrategias de destete ventilatorio precoz y el uso temprano de modalidades parciales, debieran constituir los pilares de su prevención.

(Palabras clave: ventilación mecánica, diafragma, atrofia muscular, estrés oxidativo).

Rev Chil Pediatr 2014; 85 (4): 491-498

\section{Introducción}

La Ventilación Mecánica (VM) es una terapia de soporte vital que busca mantener una adecuada ventilación alveolar y un intercambio gaseoso efectivo en pacientes críticamente enfermos. Sus indicaciones más frecuentes son la falla respiratoria aguda, el compromiso de conciencia y la exacerbación de una patología respiratoria crónica ${ }^{1}$. La VM es necesaria para la sobrevida en estos pacientes, pero su prolongación está asociada a diversas complicaciones, tales como neumonía, lesión pulmonar y disfunción diafragmática ${ }^{2-5}$. Así, el retiro de la VM debe ser precoz, mediante una estrategia programada denominada destete ventilatorio, que consiste en la reducción gradual de la asistencia ventilatoria, a la vez que el paciente asume progresivamente el trabajo ventilatorio. Este proceso finaliza con el retiro del tubo endotraqueal, evento conocido como extubación ${ }^{6}$.

En general, el período destinado al destete representa entre el $40-50 \%$ de la duración total de la VM, sin embargo, algunos pacientes fracasan, aumentando la estadía hospitalaria, costos en salud y mortalidad ${ }^{7}$. Reportes recientes sugieren que la VM controlada (VMC), la cual asume completamente el trabajo ventilatorio, pudiera contribuir al fracaso de la extubación, debido a que induce en el diafragma tanto alteraciones estructurales (atrofia y daño muscular) como funcionales (disminución de la fuerza y resistencia a la fatiga). Esta condición ha sido denominada disfunción diafragmática inducida por VM (DDIVM) $)^{4,5,8,9}$.
Los efectos de la VMC sobre la estructura y función del diafragma, así como los mecanismos moleculares involucrados en la DDIVM serán el objetivo de esta revisión. Además, discutiremos las posibles implicancias clínicas de estos nuevos hallazgos, evaluando herramientas que nos permitan aproximarnos al diagnóstico y potenciales intervenciones capaces de atenuar esta complicación.

\section{Efectos de la VMC sobre la estructura del diafragma}

La atrofia muscular por desuso es una condición que, en ausencia de enfermedad, puede producirse debido a la inmovilidad, sedentarismo, envejecimiento, microgravidez o enfermedades crónicas ${ }^{10}$. Sus manifestaciones incluyen la disminución en el diámetro de las fibras musculares y en el contenido de proteínas musculares (desbalance síntesis/degradación), así como la disminución en la capacidad de generar fuerza y resistencia a la fatiga. Debido a la dificultad para establecer los mecanismos responsables de esta complicación en humanos, los estudios en animales han permitido dilucidar este fenómeno, identificándose que la atrofia del diafragma durante la VM ocurre tempranamente, previo al desarrollo de atrofia de musculatura periférica, también inactiva durante la $\mathrm{VM}^{10-13}$. De hecho, los niveles de atrofia presente en el diafragma al cabo de $12 \mathrm{~h}$ son comparables a los observados en el músculo sóleo a las 96 h de descarga ${ }^{10,14}$. Más 
aún, la atrofia inducida por VM ha mostrado ser mayor a la reportada en el diafragma denervado ${ }^{15,16}$.

La programación de los parámetros de la VMC también pudiera tener relevancia, donde la presión positiva al final de la espiración (PEEP) podría acelerar el proceso de atrofia, ya que posicionaría al diafragma inactivo en situación de acortamiento ${ }^{9,17,18}$. Este fenómeno es concordante con estudios que indican un incremento en los niveles de atrofia en músculos periféricos inmovilizados en posición de acortamiento ${ }^{19,20}$.

Ultra-estructuralmente, en la DDIVM se aprecia disrupción de miofibrillas, incremento en el número de vacuolas en el sarcoplasma y disrupciones en la membrana mitocondrial, especulándose que la activación de la calpaína secundaria al estrés oxidativo podrían contribuir a estas alteraciones ${ }^{5}$.

\section{Efectos de la VMC sobre la función del diafragma}

El diafragma es el único músculo esquelético esencial para la vida, actuando junto a los pulmones para lograr un intercambio gaseoso efectivo. Desde el punto de vista mecánico, su contracción produce una presión negativa que incrementa la dimensión céfalo-caudal de la caja torácica. Este movimiento genera un aumento de la presión intraabdominal y disminución de la presión pleural, diferencia denominada presión transdiafragmática (Pdi), la cual representa la fuerza del músculo diafragma ${ }^{21,22}$. Estudios in vivo en diversas especies animales, han mostrado que la Pdi, medida a través de la estimulación del nervio frénico, experimenta una reducción temprana y progresiva, la que ocurre a nivel del acoplamiento excitación/contracción y de la depolarización del sarcolema, es decir, en la misma fibra muscular ${ }^{18,23-25}$. Los estudios in vitro han confirmado estos resultados, evidenciando que tanto en contracción aislada como tetánica, las fibras diafragmáticas experimentan una reducción de su fuerza isométrica máxima en un 30-50\% $\%^{26-28}$.

Los efectos de la VMC sobre la resistencia a la fatiga diafragmática son controversiales.
Anzueto et al mostraron una disminución de la resistencia a la fatiga de un $36 \%$, tras una sobrecarga inspiratoria resistiva en babuinos sometidos a VMC durante 11 días $^{23}$, pero esto no ha sido corroborado en otros estudios, donde emplearon tiempos más breves ${ }^{17,18,29}$. De este modo, la disminución de la resistencia a la fatiga diafragmática pudiera ser un efecto tardío durante la VM.

La relación fuerza-velocidad del diafragma, medida que cuantifica la velocidad máxima de acortamiento, tiene un comportamiento inesperado en VMC. La velocidad máxima de acortamiento experimenta un incremento tras 1 a 3 días de VMC. Este incremento se interpreta como un mecanismo compensatorio para preservar la potencia del diafragma debido al déficit de fuerza ${ }^{30}$.

De este modo, la atrofia por desuso diafragmática induce cambios disímiles en la fuerza, resistencia a la fatiga y velocidad de contracción.

\section{Efectos de la VMC sobre la mecano- transducción del músculo diafragma}

Las células son capaces de detectar estímulos mecánicos, transformándolos en señales bioquímicas, proceso comúnmente conocido como mecanotransducción. Si el estímulo mecánico es excesivo, la mecanotransducción puede desencadenar una respuesta nociva. En términos generales, la atrofia muscular por desuso se manifiesta a través de una disminución del contenido muscular de proteínas, debido a una reducción de su síntesis o un aumento de su degradación (proteólisis) ${ }^{10}$. Shanely et al reportaron una reducción en la síntesis de proteínas musculares mixtas y de las cadenas pesadas de miosina tras seis horas de $\mathrm{VMC}^{13}$, mientras Futier et al, observaron una reducción progresiva en la síntesis proteica, que antecedió al aumento de su degradación ${ }^{31}$. Las vías moleculares relacionadas con la proteólisis han sido más profundamente estudiadas, observándose que los sistemas proteolíticos intracelulares calpaína, caspasa y ubiquitinaproteosoma incrementan su actividad diafragmática durante la $\mathrm{VM}^{11,12,32}$. La activación de 
calpaína y caspasa-3 tienen la capacidad de disociar el complejo actina-miosina, dando paso a la actividad proteolítica de ubiquitinaproteosoma $^{5,9,10}$ (figura 1).

La calpaína es una cisteína proteasa cuya activación depende de los niveles citoplasmáticos de calcio y de la concentración intracelular de su inhibidor. Su actividad proteolítica no se dirige directamente a las proteínas contráctiles, sino que a las proteínas estructurales que mantienen el ensamble de la sarcómera, lo que propicia el desemsamblaje de los miofilamentos ${ }^{10}$. Si bien aún no es bien conocido el estímulo primario que desencadena la activación de la calpaína en el diafragma, la disminución de la expresión de la enzima SERCA (Sarcoplasmic/endoplasmic reticulum calcium ATPase), responsable de la remoción de calcio del sarcoplasma, podría contribuir a su activación ${ }^{33}$. Por su parte, la caspasa-3, cuya actividad es conocida como un marcador de apoptosis celular, es una endoproteasa que degrada numerosas proteínas musculares y juega un rol importante en condiciones de atrofia muscular por desuso ${ }^{12}$. Su activación específica es compleja e involucraría diversas rutas de seña- lización interconectadas, siendo precedida de la activación de otras caspasas, e incluso de la misma calpaína ${ }^{10,34}$.

Existen varios reportes que vinculan el aumento de la actividad proteolítica con el estrés oxidativo en el diafragma durante la $\mathrm{VMC}^{31,34-}$ 37. Whidden et al demostraron la activación de los sistemas proteolíticos mencionados a través de una señal corriente arriba que involucra al estrés oxidativo ${ }^{34}$. El estrés oxidativo se produce por un desbalance entre la producción de radicales libres y la capacidad antioxidante neutralizadora a nivel celular ${ }^{10}$. El músculo esquelético habitualmente produce especies reactivas de oxígeno (ROS) y de nitrógeno (RNS), tanto durante la contracción como en reposo. Estos actuarían sobre proteínas, lípidos y ácidos nucleicos, produciendo tanto alteraciones estructurales como funcionales en el músculo esquelético en desuso. Se ha identificado un incremento en la oxidación de proteínas (cuantificada como formación de proteínas carboniladas), de la peroxidación de lípidos (medida como formación de hidroxiperóxidos y modificación de proteínas por 4-hidroxi2-nonenal) y la disminución de sistemas enzi-

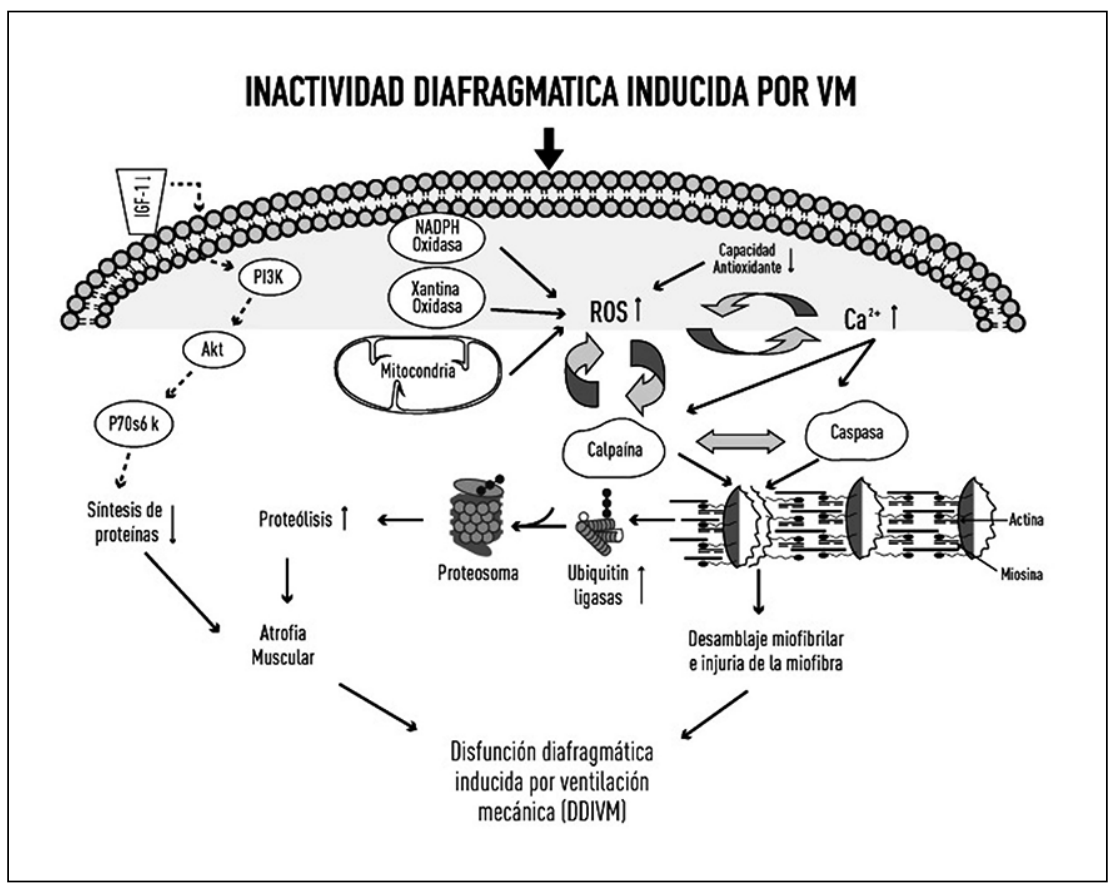

Figura 1. Rutas de señalización molecular en disfunción diafragmática inducida por ventilación mecánica. IGF-1: Factor de crecimiento insulínico tipo 1; PI3K: Fosfatidil-inositol 3 kinasa; p70 ${ }^{\mathrm{s} 6 \mathrm{k}}$ : p70S6 kinase; NADPH: Nicotinamida adenina dinucleótido fosfato; ROS: especies reactivas de oxígeno; $\mathrm{Ca}^{2+}$ : calcio. 
máticos y no-enzimáticos antioxidantes neutralizadores ${ }^{34-37}$. Zergeroglu et al evidenciaron que el incremento en los niveles de proteínas carboniladas, así como de los hidroxiperóxidos lipídicos en el diafragma, se producía al cabo de 6 h de VM, y que los efectos del estrés oxidativo podrían afectar la expresión de proteínas contráctiles ${ }^{35}$. Esto no es sorprendente si consideramos estudios en otros músculos estriados. La primera evidencia sobre la contribución del estrés oxidativo en la atrofia por desuso del músculo sóleo fue reportada hace ya casi 2 décadas por Kondo et al, donde la inmovilización muscular estaba asociada a lesión oxidativa ${ }^{38}$. Posteriormente, los mismos autores observaron que la administración de antioxidantes exógenos, como la vitamina $\mathrm{E}$, mitigaban los efectos asociados a la atrofia muscular por desuso en aproximadamente un $20 \%{ }^{39}$. De modo semejante, un estudio reciente reportó que la administración de un análogo de la vitamina $\mathrm{E}$, atenúa la proteólisis asociada al estrés oxidativo ${ }^{37}$.

Últimamente se ha evidenciado que el estrés oxidativo también jugaría un rol en la reducción de la síntesis proteica diafragmática. Específicamente, la disminución de la actividad de Akt (proteína kinasa B), cuya función está asociada a la mantención del tamaño de la fibra muscular, deriva en una reducción de la síntesis proteica y un aumento en la proteólisis. La fosforilación de Akt está precedida de la activación de IGF-1 (Factor de crecimiento insulínico tipo 1) y de la PI3K (fosfatidilinositol 3 kinasa). Además, la inactivación de Akt activa a FoxO (forkhead box O), la que a su vez activa los factores de transcripción asociados a la proteólisis (ubiquitin-ligasas) ${ }^{40}$. En este sentido, McClung et al demostraron que 6 y $18 \mathrm{~h}$ de VMC produjeron una regulación inhibitoria en la ruta de señalización molecular IGF-1-PI3K-Akt. No obstante, también observaron que esta regulación negativa se produjo a pesar de la atenuación de la atrofia diafragmática mediada por antioxidantes, lo que nos indica que esta ruta de señalización molecular es necesaria en el desarrollo de atrofia diafragmática inducida por $\mathrm{VM}^{40}$.

En otro estudio, McClung et al observaron una reducción en la fosforilación de la proteína p $70^{\mathrm{s} 6 \mathrm{k}}$ (proteína efectora en la ruta de síntesis de proteína a nivel ribosomal) en el diafragma de ratas al cabo de $6 \mathrm{~h}$ de $\mathrm{VMC}$, sugiriendo que la regulación negativa en la etapa de iniciación de la traducción en la síntesis proteica podría ser el mecanismo responsable de la alteración en la síntesis de proteínas ${ }^{41}$.

Aún cuando existe asociación entre estrés oxidativo y DDIVM, no hay certeza del mecanismo exacto que explique el rol de los radicales libres en los procesos relacionados con el metabolismo proteico en el diafragma. Un mecanismo propuesto es el aumento en la peroxidación lipídica, medido como producción del aldehído reactivo 4-hidroxi-2-nonenal, identificado bajo condiciones de sepsis y de sobrecarga muscular resistiva ${ }^{42}$. Este compuesto reduce la actividad de la bomba $\mathrm{Ca}^{+2}$-ATPasa en la membrana plasmática de los neutrófilos, favoreciendo la acumulación de $\mathrm{Ca}^{+2}$ en el citoplasma celular ${ }^{43}$. Este fenómeno podría extrapolarse a la fibra muscular diafragmática, así explicaríamos la activación de la calpaína y el aumento de la degradación de proteínas musculares $5,9,10$.

El impacto del estrés oxidativo en el diafragma durante la VMC no sólo se produciría por un aumento en la producción de ROS sino también por una reducción de la capacidad antioxidante de la fibra muscular ${ }^{36}$. Por otra parte, diversos estudios ${ }^{44-47}$ han intentado revelar la fuente primaria de producción de ROS en el diafragma durante la VMC, sin embargo, hasta ahora lo que sabemos es que la contribución de la NADPH oxidasa (Nicotinamida adenina dinucleótido fosfato) y de la NOS (óxido nítrico sintetasa) es bastante baja ${ }^{44,45}$. Por otra parte, se ha observado que la inhibición de la xantina oxidasa, favorece levemente la contractibilidad, pero no logra evitar la atrofia diafragmática $^{46}$. Por último, Kavazis et al mostraron en un estudio reciente que la mitocondria diafragmática de ratas libera gran cantidad de ROS durante la VMC y además se evidenció daño oxidativo mitocondrial en lípidos y proteínas musculares ${ }^{47}$. Es relevante mencionar que no existe evidencia de una infiltración de células inflamatorias o de la expresión génica de citoquinas pro-inflamatorias locales en el diafragma durante la $\mathrm{VMC}^{48-49}$. 


\section{Antecedentes en humanos e implicancias clínicas}

En humanos, la DDIVM no está del todo dilucidada, debido a limitaciones en el uso de técnicas de medición y a la presencia de diversos factores que podrían influir en las mediciones (vide infra) ${ }^{22}$. Knisely et al realizaron estudios histopatológicos post-mortem en 13 recién nacidos sometidos a VM por más de 12 días, evidenciando una disminución en el área de sección transversal diafragmática, cambio no observado en pacientes ventilados por menos de 7 días $^{50}$. En concordancia, Levine et al mostraron una marcada reducción en el área de sección transversal de fibras lentas y rápidas en el diafragma de 14 pacientes sometidos a 18-69 h de VM, en contraste con pacientes ventilados durante $2-3 \mathrm{~h}$ asociando directamente la atrofia diafragmática y la expresión génica de ubiquitina-ligasas ${ }^{51}$.

La medición clínica de las propiedades contráctiles de los músculos ventilatorios es compleja, debido a su localización y la dificultad para aislar la participación de los músculos involucrados. Técnicas específicas para medir la fuerza de los músculos ventilatorios se han descrito para el diafragma, esternocleidomastoideo y abdominales, mientras que la medición de la fatiga, definida como la reducción de la capacidad de generar fuerza bajo condiciones de sobrecarga, se ha centrado principalmente en el diafragma ${ }^{22}$. Ambas mediciones requieren de la participación activa del sujeto y se obtienen a través de la capacidad de generar presión durante una maniobra máxima voluntaria (Presión inspiratoria máxima, $\mathrm{PI}_{\max }$ ), así como a través de la relación entre la presión inspiratoria de cada respiración y la $\mathrm{PI}_{\max }$ bajo condiciones de sobrecarga aguda ${ }^{52}$. Sin embargo, durante la VM, estas mediciones han sido cuestionadas por su escasa confiabilidad, ya que dependen de la cooperación del paciente, y por la pobre asociación con el resultado final del destete ventilatorio ${ }^{53,54}$. Recientes estudios han propuesto la estimulación bilateral anterior magnética del nervio frénico como un método confiable, que prescinde de la colaboración activa del sujeto, y que permite medir tanto fuerza como resistencia a la fatiga. Este método consiste en la estimulación magnética supramáxima de ambos nervios frénicos en la zona anterior del cuello, y requiere de la instalación de un balón gástrico y esofágico ${ }^{54,55}$. Un estudio prospectivo observacional de Hermans et al, reportó una asociación inversa entre fuerza diafragmática y permanencia en $\mathrm{VM}^{55}$.

\section{Potenciales estrategias terapéuticas}

En base al conocimiento actual, tras lograr la estabilización del paciente debiéramos considerar la utilización temprana de modalidades ventilatorias parciales y de niveles de PEEP moderados a bajos, además el empleo de estrategias de destete ventilatorio protocolizados, que permitan reducir al mínimo el tiempo bajo asistencia ventilatoria mecánica. Complementariamente, en pacientes de riesgo de desarrollar DDIVM debemos limitar la utilización de aquellos factores responsables de miopatía de paciente crítico, como esteroides sistémicos, bloqueadores neuromusculares, glicopéptidos, aminoglicósidos, depleción electrolítica, entre otros, así como prevenir o tratar tempranamente aquellos responsables de inducir polineuropatía, como hiperglicemia, sepsis y falla multiorgánica. Finalmente, futuros ensayos clínicos debieran dilucidar el potencial rol de antioxidantes, como terapia adyuvante o preventiva de la DDIVM.

\section{Conclusiones}

La disfunción diafragmática inducida por $\mathrm{VM}$ es una entidad emergente, de creciente conocimiento y potencialmente prevenible. Ésta es responsable de inducir cambios estructurales, funcionales y en vías de transducción molecular, desencadenados fundamentalmente por la sobre-expresión de vías patogénicas proteolíticas y estrés oxidativo, siendo el principal estímulo para su desarrollo, el tiempo de permanencia en VM. De este modo, el empleo de estrategias activas de destete ventilatorio precoz y el uso temprano de modalidades parciales, tras lograr su estabilización, debieran constituir los pilares de su prevención. 


\section{Referencias}

1.- Farias JA, Frutos F, Esteban A, et al: What is the daily practice of mechanical ventilation in pediatric intensive care units? A multicenter study. Intensive Care Med 2004; 30: 918-25.

2.- Chastre J, Fagon JY: Ventilator-associated pneumonia. Am J Respir Crit Care Med 2002; 165: 867-903.

3.- Slutsky AS: Ventilator-induced lung injury: from barotrauma to biotrauma. Respir Care 2005; 50: 646-59.

4.- Vassilakopoulos T, Petrof BJ: Ventilator-induced diaphragmatic dysfunction. Am J Respir Crit Care Med 2004; 169: 336-41.

5.- Vassilakopoulos T: Ventilator-induced diaphragm dysfunction: the clinical relevant of animal models. Intensive Care Med 2008; 34: 7-16.

6.- Valenzuela J, Araneda P, Cruces P: Weaning from mechanical ventilation in paediatrics. State of the art. Arch Bronconeumol 2014; 50: 105-12.

7.- Boles JM, Bion J, Connors A, et al: Weaning from mechanical ventilation. Eur Respir J 2007; 29: 1033-56.

8.- Vassilakopoulos T, Zakynthinos S, Roussos C: Benchto-bedside review: weaning failure-should we rest the respiratory muscles with controlled mechanical ventilation? Crit Care 2006; 10: 204.

9.- Petrof BJ, Jaber S, Matecki S: Ventilator-induced diaphragmatic dysfunction. Curr Opin Crit Care 2010; 16 : 19-25.

10.- Powers SK, Kavazis AN, McClung JM: Oxidative stress and disuse muscle atrophy. J Appl Physiol 2007; 102: 2389-97.

11.- Shanely RA, Zergeroglu MA, Lennon SL, et al: Mechanical ventilation-induced diaphragmatic atrophy is associated with oxidative injury and increased proteolytic activity. Am J Respir Crit Care Med 2002; 166 : 1369-74.

12.- McClung JM, Kavazis AN, Deruisseau KC, et al: Caspase-3 regulation of diaphragm myonuclear domain during mechanical ventilation-induced atrophy. Am J Respir Crit Care Med 2007; 175: 150-9.

13.- Shanely RA, Van Gammeren D, Deruisseau KC, et al: Mechanical ventilation depresses protein synthesis in the rat diaphragm. Am J Respir Crit Care Med 2004; 170: 994-9.

14.- Thomason DB, Biggs RB, Booth FW: Protein metabolism and betamyosin heavy-chain mRNA in unweighted soleus muscle. Am J Physiol Regul Integr Comp Physiol 1989; 257: R300-5.

15.- Geiger PC, Bailey JP, Zhan WZ, Mantilla CB, Sieck $G C$ : Denervation-induced changes in myosin heavy chain expression in the rat diaphragm muscle. J Appl Physiol 2003; 95: 611-9.
16.- Zhan WZ, Miyata H, Prakash YS, Sieck GC: Metabolic and phenotypic adaptations of diaphragm muscle fibers with inactivation. J Appl Physiol 1997; 82: 1145-53.

17.- Capdevila X, López S, Bernard N, et al: Effects of controlled mechanical ventilation on respiratory muscle contractile properties in rabbits. Intensive Care Med 2003; 29: 103-10.

18.- Sassoon CS, Caiozzo VJ, Manka A, Sieck GC: Altered diaphragm contractile properties with controlled mechanical ventilation. J Appl Physiol 2002; 92: 2585-95.

19.- Jarvinen MJ, Einola SA, Virtanen EO: Effect of the position of immobilization upon the tensile properties of the rat gastrocnemius muscle. Arch Phys Med Rehabil 1992; 73: 253-7.

20.- Jokl P, Konstadt S: The effect of limb immobilization on muscle function and protein composition. Clin Ortho Relat Res 1983: 222-9.

21.- Poole DC, Sexton WL, Farkas GA, Powers SK, Reid $M B$ : Diaphragm structure and function in health and disease. Med Sci Sports Exerc 1997; 29: 738-54.

22.- Polkey MI, Moxham J: Clinical aspects of respiratory muscle dysfunction in the critically ill. Chest 2001; 119: 926-39.

23.- Anzueto A, Peters JI, Tobin MJ, et al: Effects of prolonged controlled mechanical ventilation on diaphragmatic function in healthy adult baboons. Crit Care Med 1997; 25: 1187-90.

24.- Radell PJ, Remahl S, Nichols DG, Eriksson LI: Effects of prolonged mechanical ventilation and inactivity on piglet diaphragm function. Intensive Care Med 2002; 28: 358-64.

25.- Jaber S, Sebbane M, Koechlin C, et al: Effects of short vs prolonged mechanical ventilation on antioxidant systems in piglet diaphragm. Intensive Care Med 2005; 31: 1427-33.

26.- Powers SK, Shanely RA, Coombes JS, et al: Mechanical ventilation results in progressive contractile dysfunction in the diaphragm. J Appl Physiol 2002; 92: 1851-58.

27.- Yang L, Luo J, Bourdon J, Lin MC, Gottfried SB, Petrof $B J$ : Controlled mechanical ventilation leads to remodeling of the rat diaphragm. Am J Respir Crit Care Med 2002; 166: 1135-40.

28.- Gayan-Ramirez G, De Paepe K, Cadot P, Decramer M: Detrimental effects of short-term mechanical ventilation on diaphragm function and IGF-I mRNA in rats. Intensive Care Med 2003; 29: 825-33.

29.- Shanely RA, Coombes JS, Zergeroglu AM, Webb AI, Powers SK: Short-duration mechanical ventilation enhances diaphragmatic fatigue resistance but impairs force production. Chest 2003; 123: 195-201.

30.- Zhu E, Sassoon CS, Nelson R, et al: Early effects of mechanical ventilation on isotonic contractile properties 
and MAF-box gene expression in the diaphragm. J Appl Physiol 2005; 99: 747-56.

31.- Futier E, Constantin JM, Combaret L, et al: Pressure support ventilation attenuates ventilator-induced protein modifications in the diaphragm. Crit Care 2008; 12: 1-9.

32.- Deruisseau KC, Kavazis AN, Deering MA, et al: Mechanical ventilation induces alterations of the ubiquitinproteasome pathway in the diaphragm. J Appl Physiol 2005; 98: 1314-21.

33.- Racz GZ, Gayan-Ramirez G, Testelmans D, et al: Early changes in rat diaphragm biology with mechanical ventilation. Am J Respir Crit Care Med 2003; 168: 297 304.

34.- Whidden MA, Smuder AJ, Wu M, Hudson MB, Nelson $W B$, Powers $S K$ : Oxidative stress is required for mechanical ventilation-induced protease activation in the diaphragm. J Appl Physiol 2010; 108: 1376-82.

35.- Zergeroglu MA, McKenzie MJ, Shanely RA, Van Gammeren D, Deruisseau KC, Powers SK: Mechanical ventilation-induced oxidative stress in the diaphragm. J Appl Physiol 2003; 95: 1116-24.

36.- Falk DJ, Deruisseau KC, Van Gammeren DL, Deering MA, Kavazis AN, Powers SK: Mechanical ventilation promotes redox status alterations in the diaphragm. $\mathrm{J}$ Appl Physiol 2006; 101: 1017-24.

37.- Betters JL, Criswell DS, Shanely RA, et al: Trolox attenuates mechanical ventilation-induced diaphragmatic dysfunction and proteolysis. Am J Respir Crit Care Med 2004; 170: 1179-84.

38.- Kondo H, Nakagaki I, Sasaki S, Hori S, Itokawa Y: Mechanism of oxidative stress in skeletal muscle atrophied by immobilization. Am J Physiol Endocrinol Metab 1993; 265: E839-44.

39.- Kondo H, Nishino K, Itokawa Y: Hydroxyl radical generation in skeletal muscle atrophied by immobilization. FEBS Lett 1994; 349: 169-72.

40.- McClung JM, Kavazis AN, Whidden MA, et al: Antioxidant administration attenuates mechanical ventilationinduced rat diaphragm muscle atrophy independent of protein kinase B (PKB Akt) signalling. J Physiol 2007; 585: 203-15.

41.- McClung JM, Whidden MA, Kavazis AN, Falk DJ, Deruisseau KC, Powers SK: Redox regulation of diaphragm proteolysis during mechanical ventilation. Am J Physiol Regul Integr Comp Physiol 2008; 294: R160817.

42.- Hussain SN, Matar G, Barreiro E, Florian M, Divangahi $M$, Vassilakopoulos $T$ : Modifications of proteins by 4-hydroxy-2-nonenal in the ventilatory muscles of rats. Am J Physiol Lung Cell Mol Physiol 2006; 290 : L996-1003.
43.- Siems W, Capuozzo E, Lucano A, Salerno C, Crifo C: High sensitivity of plasma membrane ion transport ATPases from human neutrophils towards 4-hydroxy-2,3-trans-nonenal. Life Sci 2003; 73: 2583-90.

44.- McClung JM, Van Gammeren D, Whidden MA, et al: Apocynin attenuates diaphragm oxidative stress and protease activation during prolonged mechanical ventilation. Crit Care Med 2009; 37: 1373-9.

45.- Van Gammeren D, Falk DJ, Deering MA, Deruisseau $K C$, Powers SK: Diaphragmatic nitric oxide synthase is not induced during mechanical ventilation. J Appl Physiol 2007; 102: 157-62.

46.- Whidden MA, McClung JM, Falk DJ, et al: Xanthine oxidase contributes to mechanical ventilation-induced diaphragmatic oxidative stress and contractile dysfunction. J Appl Physiol 2009; 106: 385-94.

47.- Kavazis AN, Talbert EE, Smuder AJ, Hudson MB, Nelson WB, Powers $S K$ : Mechanical ventilation induces diaphragmatic mitochondrial dysfunction and increased oxidant production. Free Radic Biol Med 2009; 46: 84250.

48.- Van Gammeren D, Falk DJ, DeRuisseau KC, Sellman $J E$, Decramer M, Powers SK: Reloading the diaphragm following mechanical ventilation does not promote injury. Chest 2005; 127: 2204-10.

49.- DeRuisseau KC, Shanely RA, Akunuri N, et al: Diaphragm unloading via controlled mechanical ventilation alters the gene expression profile. Am J Respir Crit Care Med 2005; 172: 1267-75.

50.- Knisely AS, Leal SM, Singer DB: Abnormalities of diaphragmatic muscle in neonates with ventilated lungs. J Pediatr 1988; 113: 1074-7.

51.- Levine S, Nguyen T, Taylor $N$, et al: Rapid disuse atrophy of diaphragm fibers in mechanically ventilated humans. N Engl J Med 2008; 358: 1327-35.

52.- Moxham J, Goldstone J: Assessment of respiratory muscle strength in the intensive care unit. Eur Respir J 1994; 7: 2057-61.

53.- Multz AS, Aldrich TK, Prezant DJ, Karpel JP, Hendler $J M$ : Maximal inspiratory pressure is not a reliable test of inspiratory muscle strength in mechanically ventilated patients. Am Rev Respir Dis 1990; 142: 529-32.

54.- Watson AC, Hughes PD, Louise Harris M, et al: Measurement of twitch transdiaphragmatic, esophageal, and endotracheal tube pressure with bilateral anterolateral magnetic phrenic nerve stimulation in patients in the intensive care unit. Crit Care Med 2001; 29: 1325-31.

55.- Hermans G, Agten A, Testelmans D, Decramer M, Gayan-Ramírez $G$ : Increased duration of mechanical ventilation is associated with decreased diaphragmatic force: a prospective observational study. Crit Care 2010; 14: R127. 